\title{
Prior Food-Induced Allergic Gut Inflammation Leading To Multiple Food Allergies in C57Bl/6 Mice
}

\author{
Airton Pereira e Silva ${ }^{1}$, Barbara Marmello ${ }^{1}$, Isabelle Guimarães ${ }^{2}$, and Gerlinde Teixeira ${ }^{1}$ \\ ${ }^{1} \mathrm{UFF}$ \\ ${ }^{2}$ Universidade Federal Fluminense
}

August 4, 2020

\begin{abstract}
BACKGROUND: Food allergies are usually managed by food avoidance. Hidden allergens in food, due to cross-contamination and/or allergenic additives added during production, place an important concern in today's increasing food allergy cases worldwide. Previous studies showed that introduction of new food components, in an inflamed intestine, results in sensitization to this food. Thus, our aim was to evaluate the kinetics of multiple food allergy induction. METHODS: Adult male C57BL/6 mice were divided into five groups, four of which were submitted to an intestinal inflammation induction protocol to peanuts. Egg white (OVA) diluted 1:5 v/v in distilled water was instilled by gavage 6h-before (EXP-1), concomitant (EXP-2) and 6h-after (EXP-3) the onset of the peanut challenge diet. Positive control (POS CONT) and NEG CONT received saline per gavage. Finally, animals were challenged with subcutaneous injections of OVA. RESULTS: No changes in diet intake were observed. Anti-OVA total IgG antibody titers significantly increased in EXP-2. Flow cytometry revealed significant decrease in CD4+CD25+Foxp3+ and significant increase in TCD8+ in EXP-2. Histomorphometrically, EXP-2 and EXP-3 were classified as Infiltrative and Partial Destruction stages. EXP-1 was classified as Infiltrative, while POS CONT was classified as Partial Destruction. NEG CONT was classified as Normal. CONCLUSION: The introduction of a new food only a few hours before the initiation of a gut inflammation is able to induce oral tolerance, however the introduction of a new dietary protein concomitant to the onset or during an ongoing gut inflammation may induce multiple allergies.
\end{abstract}

\section{Introduction}

Food allergy, defined as an impaired reaction to food proteins by the immune system, has become a major public health concern in recent years ${ }^{1,2}$. Symptoms vary drastically from mild to extreme (anaphylaxys), a life-threatning condition ${ }^{1,3}$. Atopic patients, with genetic predisposition, activate specific immune reactions to allergens, some of which maintain or even increase their allergenicity even when cooked ${ }^{1}$.

Several papers in the literature have shown throughtout the last decade an increase in food allergy diagnosis around the globe, mostly in Western countries ${ }^{4-8}$. In the United States, the estimated prevalence of food allergies is $3.9 \%$ to $8 \%$ in children and $6.6 \%$ to $10 \%$ in adults ${ }^{6}$. The reasons for this surge are not yet fully understood, although some researches have linked the increase of food allergies with lack of microbial exposure during infancy, resulting in immunological reactions to benign proteins, such as foods ${ }^{9}$.

Food allergies negatively impact not only the patients's health, but also their familie's life quality ${ }^{10}$, as everyday activities like grocery shooping, cooking and dining out can be affected ${ }^{9}$. Therefore, it is absolutely essential to develop novel methods to prevent immunological reactions altogether. Treatments often include oral immunotherapy ${ }^{11}$, which is an attempt to nurture the immune system by progressively increasing doses of the allergen orally until reaching a target dose that is then ingested regularly ${ }^{12,13}$. Nevertheless, this therapy can trigger anaphylatic reactions, hence requiring a medical facility ${ }^{14}$. 
The amount of allergen taken cannot predict the severity of the symptoms ${ }^{3,15}$, this means that life-threatning symptons may develop due to even traces of the allergen ${ }^{16,17}$. One important source of unknown allergenic components are food addictives used by the industry in processed products, derived from both vegetable and animal proteins such as soy, egg white, milk, etc ${ }^{18,19}$. The golden standard for food allergy therapy is to completely avoid the offending protein ${ }^{20}$. However, one may have trouble avoiding unexpected contacts, especially children, to hidden allergens ${ }^{21}$. During processing, such as in mills, cross-contamination may occur resulting in hidden allergens that may not appear in the ingredients list ${ }^{3,22}$. Consequently, it is possible that one ingests food containing not only tolerated proteins, but also food allergens that can provoke immune reactions. Thus, our aim was to evaluate the kinetics of multiple food allergies induction and progression.

\section{Material and Methods}

\section{Animals}

C57Bl/6 adult (70 days old) inbred male mice bred at the Animal Facility of the Federal Fluminense University (Niteroi, RJ, Brazil) were given free access to mouse chow and acidified water ( $\mathrm{pH} 2.5)$. They were kept in polypropylene cages with stainless steel covers (temperature of $22^{\circ} \mathrm{C}, \sim 60 \%$ humidity and $12 \mathrm{~h} \mathrm{light} / 12 \mathrm{~h}$ dark cycle) and were not kept either under specific pathogens free or germ-free conditions.

To ensure animals were healthy, they were monitored for one month prior to the experiment. Body weight was assessed weekly along with visual inspection of disease symptoms, like labored respiration or pilar erecti 23,24. This work was approved by the Institutional Animal Care and Use Committee (official name: Animal Research Ethics Committee - permit number 733/2016) and follows the national animal welfare committee and the ARRIVE guidelines ${ }^{25}$.

\section{Food}

Commercial mouse chow (Nuvilab CR1 - NUVILAB-NUVITAL ${ }^{\circledR}$, Sao Paulo, Brazil) without egg white or peanut proteins was offered conforming to the animal facility routine. According to the experimental timeline animals received challenge diets either composed of peanutin natura (P-CD) or inhouse OVA challenge diet (O-CD) according to AIN-93 (report of the American Institute of Nutrition that establishes standards for nutritional studies with experimental rodents ${ }^{26}$ ). Food intake was measured three times a week and the mean caloric intake was calculated per gram of body weight per cage and expressed as $\mathrm{mg} / \mathrm{gbw}$.

\section{Antigenic proteins and Immunization protocols}

Animals were immunized subcutaneously twice, (21-day interval), with $100 \mu \mathrm{g}$ of the specific protein: peanut protein extract $(\mathrm{PPE})^{27}$ or OVA with (primary) or without (booster) $1 \mathrm{mg}$ of alum adjuvant $\left[\mathrm{Al}(\mathrm{OH})_{3}\right]$.

\section{Gavages}

According to the experimental protocol timeline (Figure 1) animals received $0.5 \mathrm{~mL}$ of either $20 \mathrm{mg} / \mathrm{mL}$ OVA solution (Sigma-Aldrich, Darmstadt, Germany) or physiological saline.

\section{Induction of the antigen-specific inflammatory gut reaction}

P-CD or O-CD were used for 60 days to induce antigen specific inflammatory gut reaction according to the experimental timeline after PPE or OVA immunization. CONT groups continued to receive commercial mouse chow as previously described ${ }^{28-30}$.

\section{Bleeding}

Animals were bled $200 \mu$ f from the retroorbital plexus 14 days after each immunization and at the end of both challenge diet periods. The sera were stored at $-20^{\circ} \mathrm{C}$ until analyses.

\section{Determination of Ab levels}

In-house serial dilution enzyme-linked immunosorbent assays (ELISA) were performed to detect specific IgG. Briefly, 96-well microplates (Alfa, Sao Paulo, Brazil) were coated with $4 \mu \mathrm{g}$ of antigen (PPE or OVA). 
A 3-fold serial dilution of each serum (starting at 1/100 v/v) was performed. Goat HRP-anti-mouse IgG (Sigma-Aldrich, Darmstadt, Germany) was used as informed by manufacturer and reactivity was revealed with OPD (Sigma-Aldrich, Darmstadt, Germany) following specifications. Results - arbitrary units of ELISA (AUE) - correspond to the area under the dilution curve of each serum.

\section{Determination of $\mathrm{T}$ and $\mathrm{B}$ lymphocyte profile}

Mesenteric lymph nodes (MLN) of each animal were collected. Cells were washed and re-suspended at $2 \times 10^{7}$ cells/mL in PBS and stained with $\alpha \mathrm{TCD} 4, \alpha \mathrm{TCD} 8, \alpha \mathrm{CD} 25, \alpha \mathrm{CD} 3, \alpha \mathrm{Foxp} 3$ and/or $\alpha \mathrm{B} 220$ according to the manufacturer's (Biolegend ${ }^{\circledR}$, San Diego, USA) instructions. After running the samples on BD ${ }^{\circledR}$-C6 Flow Cytometer (Franklin Lakes, USA), all analyses were performed in the activated lymphocyte gate. We evaluated the following lymphocytes populations: $\mathrm{TCD} 4^{+}, \mathrm{TCD} 8^{+}, \mathrm{TCD} 4^{+} \mathrm{CD} 25^{+} \mathrm{Foxp} 3^{+}$and B-B220 ${ }^{+} \mathrm{CD} 3^{-} \mathrm{CD} 25^{+}$.

\section{Histomorphometry}

Animals received an overdose of anesthetics $(60 \mathrm{mg} / \mathrm{kg}$ of Xylazine $+350 \mathrm{mg} / \mathrm{kg}$ of Ketamine, produced by Sespo Industries ${ }^{\circledR}$, Paulinia, Sao Paulo, Brazil), after which a longitudinal section was performed to expose the peritoneum. The abdominal cavity was examined to detect any macroscopic alteration. Intestinal segments were collected from each animal and were immediately fixed with $10 \%$ buffered formaldehyde, processed and stained with hematoxylin-eosin (HE). Tissue sections were scanned with Aperio ${ }^{\circledR}$ Scanscope (Leica Microsystems GmbH, Wetzlar, Germany) and then analyzed with ImageScope software (version 11.2.0.780; Leica Microsystems GmbH, Wetzlar, Germany) to quantify duodenum histological parameters. We analyzed intestinal architecture integrity, number of villi per $8000 \mu \mathrm{m}$ of intestinal tissue, mean villus area and leukocyte infiltrates. Ratios between villus height/width $(\mathrm{H} / \mathrm{W})$ and intestinal epithelial cells/intraepithelial leukocytes (IEC/IEL) were established.

We also performed the Swiss Roll in the jejunum of each animal to evaluate the integrity of the intestinal layers, global architecture of the villi, crypts and the presence of intraepithelial lymphocytes as previously described $^{31}$. Based on duodenum and jejunum parameters, animals were classified according to the intestinal inflammation index developed by our group ${ }^{32}$.

All segments of duodenum and jejunum, as well as flow cytometry samples, were collected, processed and scanned under the same light and temperature conditions to avoid changes in cells and tissues optical properties, such as light absorption and scattering ${ }^{33}$.

\section{Experimental groups and timeline}
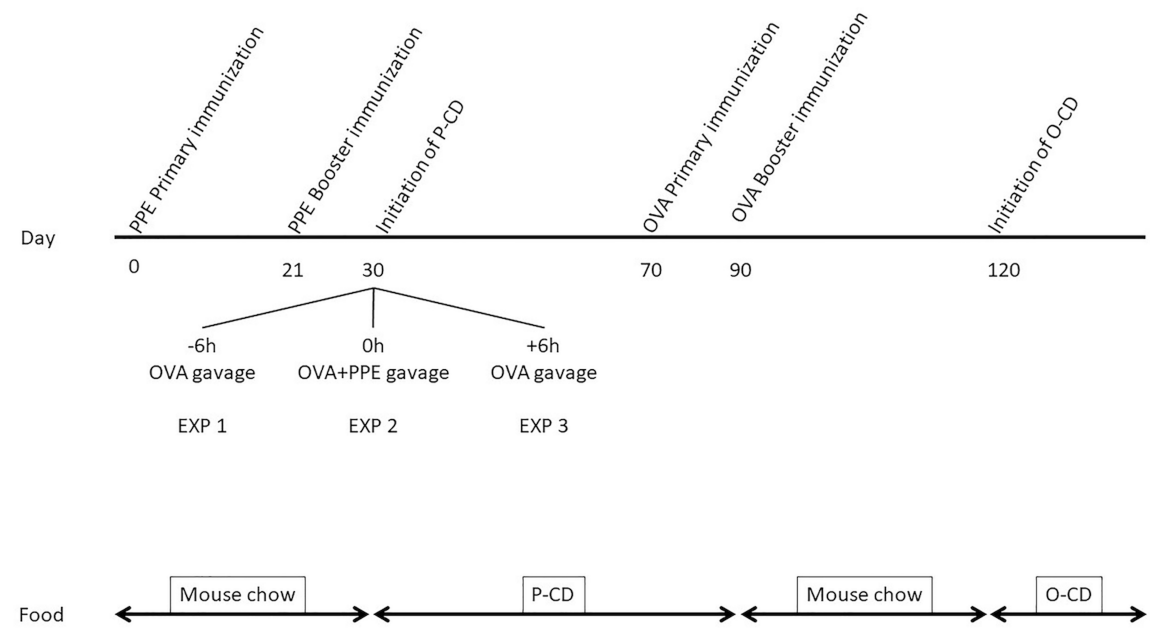

NEG CONT: sham immunized with $0.2 \mathrm{~mL}$ saline - saline gavage ( $\mathrm{n}=10 /$ group) sham immunized with 0.2 $\mathrm{mL}$ saline. 
POS CONT: immunized with 100ug of PPE, - saline gavage ( $\mathrm{n}=10 /$ group), immunized with 100ug of OVA. EXP-1: immunized with 100ug of PPE, -OVA gavage 6h prior to the introduction of peanut challenge diet ( $\mathrm{n}=10 /$ group), immunized with 100ug of OVA.

EXP-2: immunized with 100ug of PPE, - OVA gavage concomitant to the introduction of peanut challenge diet ( $\mathrm{n}=10$ /group), immunized with 100ug of OVA.

EXP-3 immunized with 100ug of PPE, - OVA gavage 6h after peanut challenge diet introduction ( $\mathrm{n}=10$ /group). immunized with 100ug of OVA.

\section{Statistical analysis}

Kolmogorov-Smirnov test was performed for normal distribution. Student $\mathrm{T}$ test when comparing two variables and one-way ANOVA with Bonferroni post-test for more than two variables were used to determine the significant difference. Tests were performed using Graphpad Prism 6 Software (Graphpad Software, Inc., La Jolla, California, United States). $\mathrm{P}<0.05$ was considered statistically significant. Results are displayed as mean+standard deviation (SD) and show data from a minimum of 10 animals for each assay. Exact $\mathrm{P}$ values are expressed in the figures.

\section{Results}

\section{Food Intake}

No significant differences were observed in kcal food intake during Peanut Allergy Induction (EXP-1: 18.46 \pm 2.94 ; EXP-2: 18.17 \pm 2.98 ; EXP-3: 19.01 \pm 3.66 POS CONT: 19.50 \pm 3.34 NEG CONT: $21.89 \pm 3.98$ Kcal). There were also no significant differences during P-CD (EXP-1: 17.59 \pm 4.93 ; EXP-2: 14.29 \pm 2.10 ; EXP-3: 17.45 \pm 3.79 ; POS CONT: 18.75 \pm 4.48 ; NEG CONT: 19.87 \pm 1.14 Kcal). However, during O-CD NEG CONT $(33.44 \pm 1.82 \mathrm{Kcal})$ and EXP-1 $(25.92 \pm 1.31 \mathrm{Kcal})$ had a significant higher consumption $(\mathrm{p}=0.0001)$ when compared to EXP-2 $(14.29 \pm 1.92 \mathrm{Kcal})$, EXP-3 $(15.95 \pm 3.41 \mathrm{Kcal})$ and POS CONT $(17.09 \pm 1.68 \mathrm{Kcal})$ (Figure S1).

\section{Anti-Peanut IgG Antibodies}

All groups inoculated with PPE had significantly higher anti-peanut total IgG antibody titers $(\mathrm{p}=0.009)$ (EXP-1: 2.18 \pm 0.37 ; EXP-2: 2.20 \pm 0.42 ; EXP-3 2.11 \pm 0.15$)$ and POS CONT: $2.47 \pm 0.45)$ when compared to NEG CONTROL (0.63 \pm 0.06$)$. EXP-2 and EXP-3 maintained significant higher IgG titers $(\mathrm{p}=0.03)$ even after weeks of PPE inoculation and P-CD when compared to EXP-1 and POS CONT (Figure 2A).

\section{Anti-OVA IgG Antibodies}

Analyzing anti-OVA total IgG antibody titers, we observed significant differences in primary inoculation ( $\mathrm{p}=0.001)$. Means of EXP (EXP-1: 1.28 \pm 0.51 ; EXP-2: $2.02 \pm 0.45$; EXP-3: $1.70 \pm 0.64)$ and POS CONT $(1.42 \pm 0.26)$ are significantly higher than NEG CONT $(0.32 \pm 0.11)$. After OVA booster inoculation, NEG CONT $(0.37 \pm 0.13)$ had significantly lower titers ( $\mathrm{p}=0.001)$ than all other groups; EXP-1 $(1.21 \pm 0.32)$ had significantly lower titers $(\mathrm{p}=0.001)$ than EXP-2 (2.51 \pm 0.33$)$, EXP-3 (2.23 \pm 0.19$)$ and POS CONT (1.73 \pm 0.19$)$. After O-CD, EXP-1 (1.22 \pm 0.25$)$ showed significantly lower titers ( $\mathrm{p}=0.01)$ than EXP-2 $(2.21 \pm 0.40)$, EXP-3 $(2,21 \pm 0.43)$ and POS CONT (1.96 \pm 0.36$)$. NEG CONT $(0.32 \pm 0.17)$ continued presenting significantly lower titers $(\mathrm{p}=0.01)$ than all other groups (Figure $2 \mathrm{~B})$. 


\section{A}

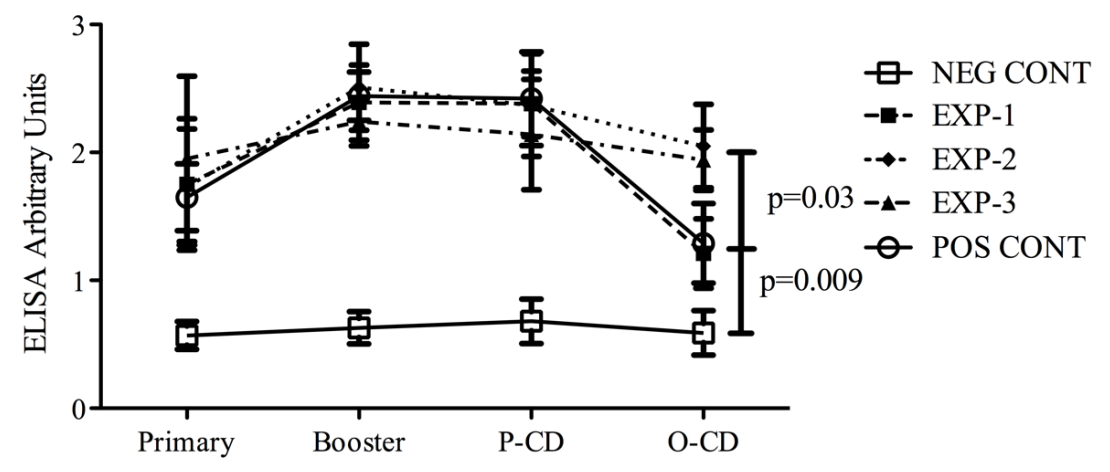

\section{B}

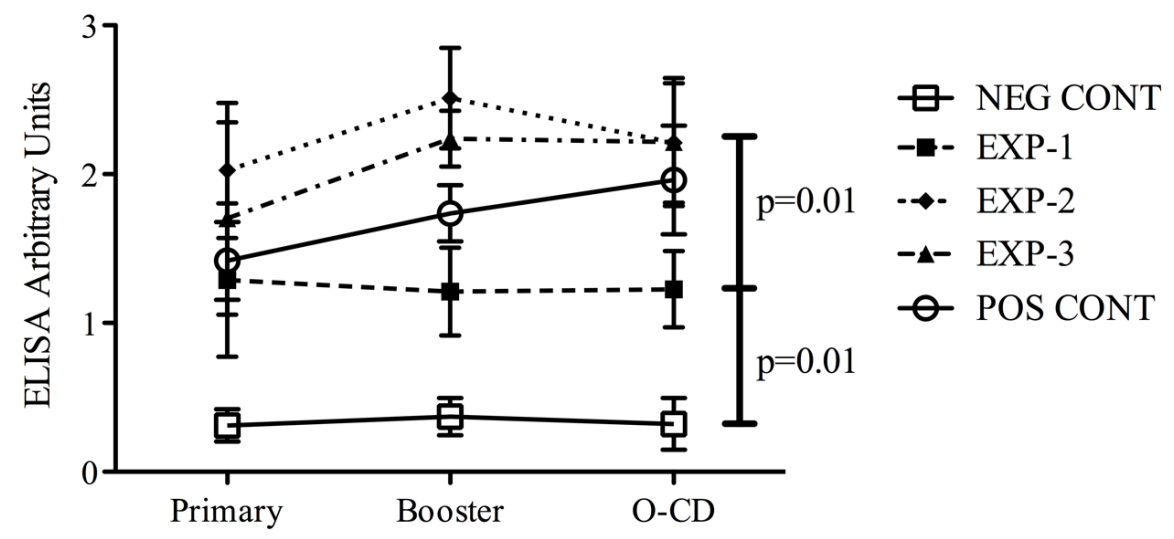

\section{Cell Phenotype in Plot}

\section{Effector Cells}

We observed a significantly higher amount of TCD $4^{+}$cells compared to TCD ${ }^{+}$cells in EXP-1 and NEG CONT due to a significant increase $(\mathrm{p}=0.0111)$ in TCD $8^{+}$observed in EXP-2 $(0.36 \pm 0.01)$, EXP-3 $(0.34 \pm 0.04)$ and POS CONT $(0.33 \pm 0.01)$ compared to the EXP-1 $(0.21 \pm 0.01)$ and NEG CONT $(0.19 \pm 0.01)$ with no significant differences in TCD4 ${ }^{+}$lymphocytes population among the groups (NEG CONT: $0.38 \pm 0.03$; EXP-1:

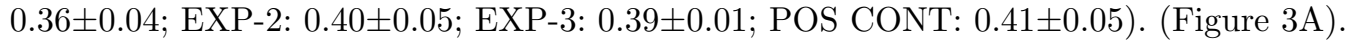

\section{Regulatory T Cells}

The percentage of TCD $4^{+} \mathrm{CD} 25^{+}$Foxp $3^{+}$cells was significantly higher $(\mathrm{p}=0.0034)$ in EXP-1 $(0.14 \pm 0.02)$ when compared to all other groups: EXP-2 (0.12 \pm 0.02$)$, EXP-3 $(0.12 \pm 0.02)$ and POS CONT $(0.11 \pm 0.02)$ and NEG CONT $(0.09 \pm 0.01)$ (Figure 3B).

\section{B Lymphocytes}

B-B220 ${ }^{+} \mathrm{CD}^{-}{ }^{-} \mathrm{CD} 25^{+}$cells percentage showed that NEG CONT $(0.06 \pm 0.01)$ and EXP-1 $(0.07 \pm 0.01)$ had a significantly smaller population $(\mathrm{p}=0.0005)$ compared to EXP-3 $(0.10 \pm 0.02)$ and POS CONT $(0.10 \pm 0.02)$. and no significant difference between EXP-1 and EXP-2 (0.09 \pm 0.01$)$ (Figure 3C). 

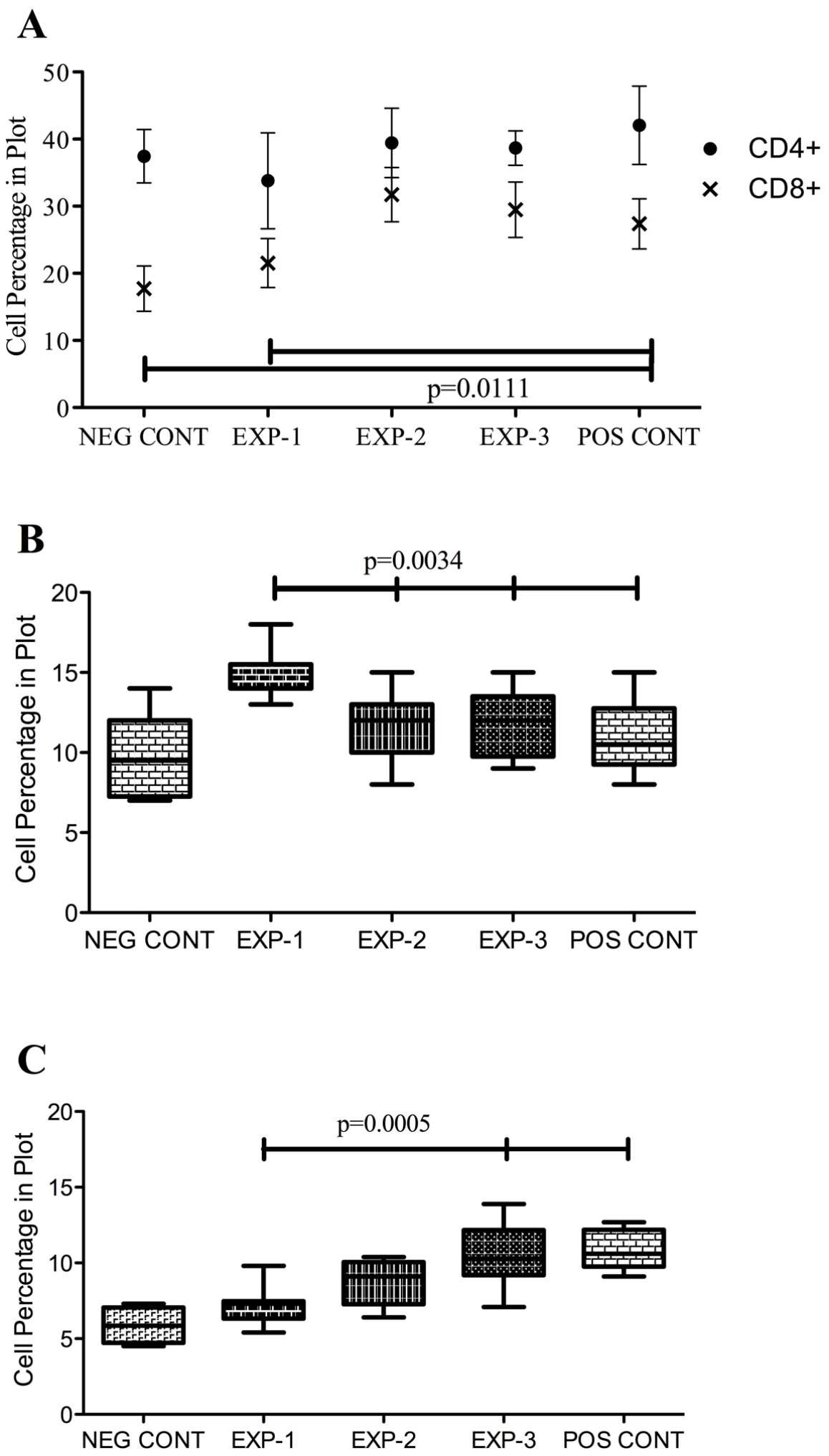

Duodenum Histomorphometry 
POS CONT (14.60 \pm 2.14$)$ had a significantly lower mean villus number per $8000 \mu \mathrm{m}$ ( $\mathrm{p}=0.0001)$ when com-

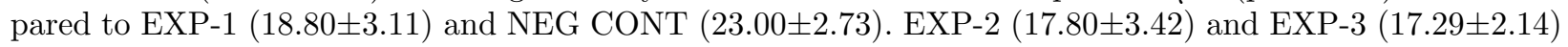
showed a significant $(\mathrm{p}=0.0001)$ lower villus number when compared to NEG CONT (Figure S2A).

Regarding villus area, EXP-1 $\left(2.2 \times 10^{4} \pm 3.6 \times 10^{3} \mu \mathrm{m}^{2}\right)$ and NEG CONT $\left(2.4 \times 10^{4} \pm 3.9 \times 10^{3} \mu \mathrm{m}^{2}\right)$ had a significantly larger mean villus area $(\mathrm{p}=0.01)$ when compared to EXP-2 $\left(1.8 \times 10^{4} \pm 2.1 \times 10^{3} \mu \mathrm{m}^{2}\right)$, EXP-3 $\left(1.7 \times 10^{4} \pm 1.7 \times 10^{3} \mu \mathrm{m}^{2}\right)$ and POS CONT $\left(1.7 \times 10^{4} \pm 1.8 \times 10^{3} \mu \mathrm{m}^{2}\right)$ (Figure S2B).

When $\mathrm{H} / \mathrm{W}$ ratio was verified, it showed that NEG CONT (4.71 \pm 0.74$)$ and EXP-1 (4.11 \pm 0.53$)$ had a significantly higher ratio when compared to EXP-2 (3.54 \pm 0.40$)$, EXP-3 (3.57 \pm 0.54$)$ and POS CONT $(3.12 \pm 0.45)$ $(\mathrm{p}=0.0036)$, which were not statistically different from each other (Figure S2C).

NEG CONT (54.60 \pm 5.32$)$ and EXP-1 (42.08 \pm 7.22$)$ IEC/IEL ratios were significantly higher $(\mathrm{p}=0.0001)$

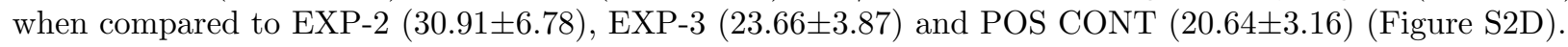

\section{Jejunum Histomorphometry}

No extensive morphological changes were observed among the groups. However, POS CONT and EXP-2 and EXP-3 presented more edema in the mucosal layer than NEG CONT and EXP-1. Reduced Mean jejunum IEC/IEL ratio of the animals that presented an inflamed duodenum (EXP-2 [17.72 \pm 5.30$]$, EXP-

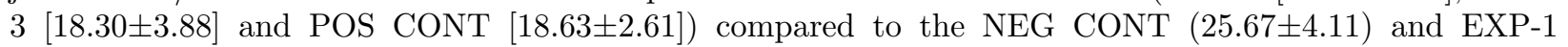
$(23.33 \pm 2.60)$ was due to a significantly larger $(\mathrm{p}=0.04)$ infiltration of intraepithelial leukocytes in the jejunum (data not shown) (Figure S2E)

\section{Classification of Intestinal Inflammatory State}

EXP-2 and EXP-3 were classified as hybrids of Infiltrative and Partial Destruction stages. EXP-1 was classified in the Infiltrative stage, while POS CONT was classified in the Partial Destruction stage. Finally, NEG CONT was classified as Normal (Table 1 and Figures 4 and 5).

Table 1 - Overview of duodenum and Jejunum parameters and classification of inflammatory state.

\begin{tabular}{|c|c|c|c|c|c|}
\hline $\begin{array}{l}\text { Parameters } \\
\text { Groups }\end{array}$ & Villus Number & $\begin{array}{l}\text { Villus Area in } \\
\mu \mathrm{m}^{2}\end{array}$ & IEC/IEL & $\begin{array}{l}\text { Jejunum } \\
\text { IEC/IEL }\end{array}$ & Classification \\
\hline NEG CONT & $23.00 \pm 2.73$ & $2.4 \times 10^{4} \pm 3.9 \times 10^{3} \quad 4.71 \pm 0.74$ & $54.60 \pm 5.32$ & $25.67 \pm 4.11$ & Normal \\
\hline EXP-1 & $18.80 \pm 3.11$ & $2.2 \times 10^{4} \pm 3.6 \times 10^{3} \quad 4.11 \pm 0.53$ & $42.08 \pm 7.22$ & $23.33 \pm 2.60$ & Infiltrative \\
\hline EXP-2 & $17.80 \pm 3.42^{*}$ & $1.8 \times 10^{4} \pm 2.1 \times 10^{3 *} 3.54 \pm 0.40^{*}$ & $30.91 \pm 6.78^{*}$ & $17.72 \pm 5.30^{*}$ & $\begin{array}{l}\text { Infiltrative/ } \\
\text { Partial } \\
\text { Destruction }\end{array}$ \\
\hline EXP-3 & $17.29 \pm 2.14^{*}$ & $1.7 \times 10^{4} \pm 1.7 \times 10^{3 *} 3.57 \pm 0.54^{*}$ & $23.66 \pm 3.87^{*}$ & $18.30 \pm 3.88^{*}$ & \\
\hline POS CONT & $14.60 \pm 2.14^{*}$ & $1.7 \times 10^{4} \pm 1.8 \times 10^{3 *} 3.12 \pm 0.45^{*}$ & $20.64 \pm 3.16^{*}$ & $18.63 \pm 2.61^{*}$ & $\begin{array}{l}\text { Partial } \\
\text { Destruction }\end{array}$ \\
\hline
\end{tabular}

H/W: height/width ratio; IEC/IEL: intestinal epithelial cells/intraepithelial leukocytes ratio. * indicates statistically different from NEG CONT. 

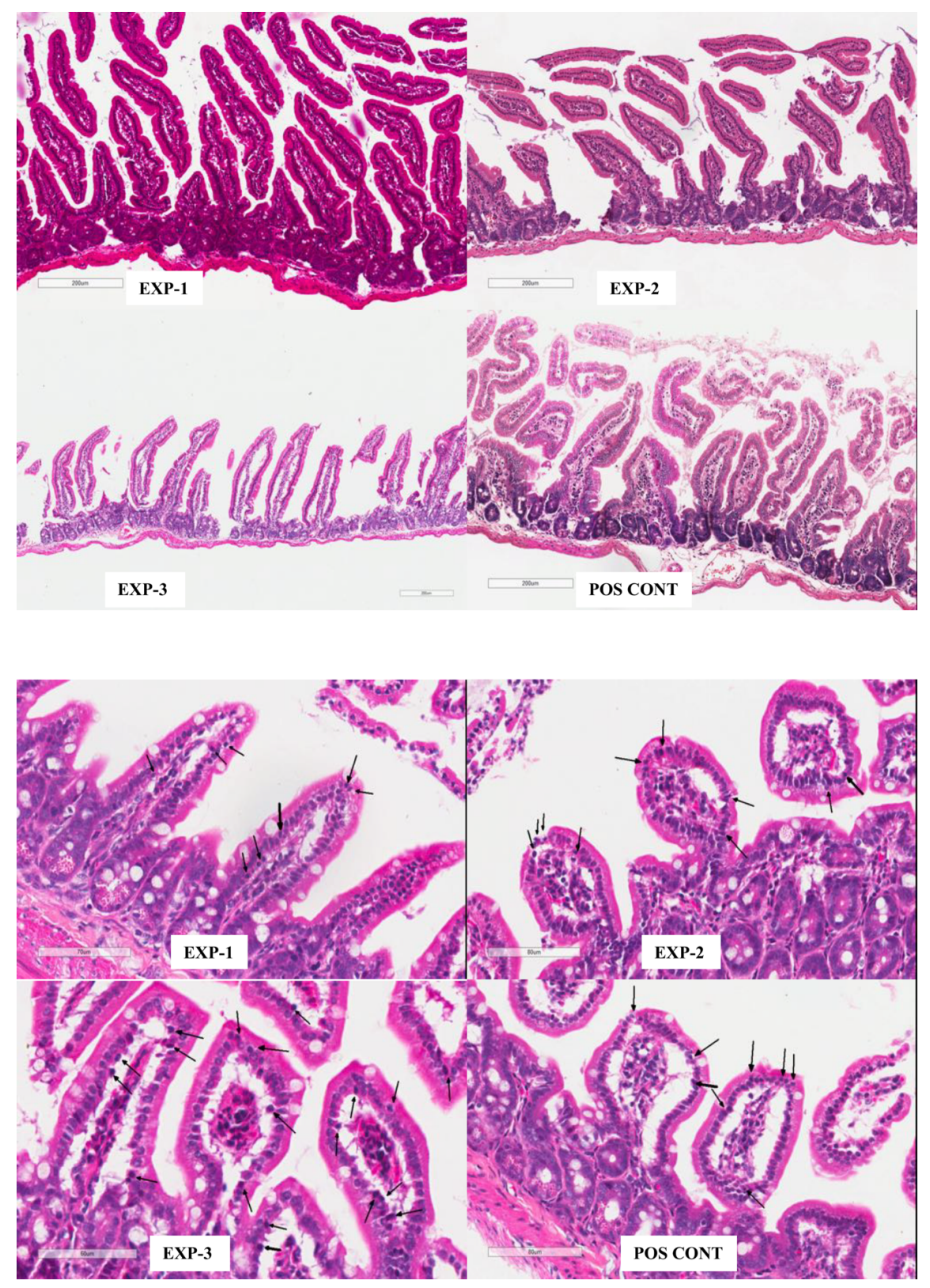

\section{Discussion}

In this work we further evaluated the timing of new food protein introduction during the induction of an antigen specific intestinal inflammation. Retrospective studies have identified several participants as intolerant to a wide variety of proteins commonly present in food ${ }^{34}$, although it is not known whether these foods are in fact more allergenic or simply the most widely consumed foods or whether the first intake of the food induced sensitization as a result of gut milieu ${ }^{35}$. An in-depth investigation in the literature showed that the distribution of food allergies varies according to populations and their cultural eating habits. For example, the top three food allergies in Brazil, although understudied, are wheat, egg, and milk ${ }^{36}$, while in Israel egg, milk, sesame and in North America shellfish, milk and peanut ${ }^{37}$. In clinical trials involving patients with allergic diseases, though changes in serological tests were observed, a total improvement of their clinical conditions is not always perceived $^{38}$. 
Food intake showed the animals in all groups consumed equivalent amounts of commercial chow and peanuts in allergy induction and P-CD periods, respectively, supporting the results found by other studies in our group $27,39,40$. The exclusive diet supply prevents animals from avoiding an allergenic food, as has already been shown for animals that have free choice between allergenic food and other food sources ${ }^{28-30}$.

Body weight evaluation demonstrated that during allergy induction all groups presented weight gain as expected for normal development, with variations consistent with animal growth and manipulation procedures to which they were submitted. Animals which only received PPE injections, commercial chow to which they were weaned and had no contact with allergens in the diet, had no changes in their intestinal mucosa. These results agree with those shown by Paschoal ${ }^{39}$ and by Tordesillas and Berin ${ }^{41}$.

A significant number of studies have shown that food allergies may develop with low expression or even absence of $\operatorname{IgE}$ antibodies ${ }^{42-45}$, thus leading to the current consensus in the literature that not all food allergies are caused by $\operatorname{IgE}$ antibodies ${ }^{46,47}$. Morita et al showed that there are three types of food allergies, from the immune response standpoint: "IgE-mediated," "combined IgE- and cell-mediated" and "non-IgEmediated $^{48}$. In our model, using C57BL/6 mice, no IgE was found, thus our model correlates to Non-IgEmediated gastrointestinal food-induced allergic disorders as also described for humans ${ }^{49,50}$.

In our study, all animals were submitted to a peanut allergy induction protocol, except NEG CONT. Thus, all groups showed high anti-peanut total IgG antibody titers, except NEG CONT. Our group has shown an increase in anti-OVA antibodies in animals that received OVA while experiencing gut inflammation because of eating an allergen-containing diet ${ }^{40}$. This present work agrees with our previous paper; however, we here show that animals that received OVA concomitant to the initiation of the challenge diet (EXP-2) presented the highest overall anti-OVA IgG titers. Animals that received OVA six hours after the initiation of the challenge diet presented the same sensitization profile and both with higher antibody titers than the subcutaneous immunization induces. On the other hand, oral introduction of the new food only six hours before the initiation of $\mathrm{P}-\mathrm{CD}$ is sufficient to induce oral tolerance. The antibody titers are comparable to NEG CONT.

In a scenario where the interaction with a new food protein is concomitant or shortly after the introduction of a protein to which one is already allergic may lead to bystander activation and not induce tolerance, even with a local low inflammatory cytokines profile ${ }^{51,52}$. Consequently, multiple allergies may be developed ${ }^{53,54}$. However, sensitization was blocked by feeding the novel food protein six hours prior to the initiation of the challenge diet. Therefore, we agree with Korte and collaborators, in which foods that share similar matrixes can cause cross responses, exacerbating immunological reactions (tolerance or sensitization) ${ }^{55}$. Though, it is not the case of this work, since the first sensitization was to a vegetable protein and the second to an animal protein that do not share a similar food matrix.

To better understand the characteristics of the immune response, both local and systemic, phenotypic analysis of cells in mesenteric lymph nodes were performed. We noticed a significant increase in the percentage of TCD8 ${ }^{+}$lymphocytes in EXP-2. According to Dahlman-Hoglund and others, IFN- $\gamma$ secreted by TCD ${ }^{+}$lymphocytes acts as a suppressor of antigen-specific TCD4 ${ }^{+}$Th2 cells in models using OVA. TCD ${ }^{+}$ lymphocytes also secrete TGF- $B$ and IL-10, which are non-antigen-specific immune response suppressor molecules and that suppression can be induced in naïve animals through the transfer of TCD8 ${ }^{+}$lymphocytes ${ }^{56,57}$.

Analyzing the percentage of TCD $4{ }^{+} \mathrm{CD} 25^{+}$Foxp $3^{+}$lymphocytes, our results showed an increase in EXP-1, which may be associated with a tolerogenic bystander suppression performed by these cells ${ }^{58,59}$. Several studies have shown that increased Foxp $3^{+}$cells may indicate tolerance induction for different allergenic proteins ${ }^{2,60,61}$. B-B220 ${ }^{+} \mathrm{CD} 3^{-} \mathrm{CD} 25^{+}$cells titers were also assessed in our study. NEG CONT, EXP-1 and EXP-2 presented lower titers than EXP-3 and POS CONT. This can be explained by the highly effector activity of B-B220 ${ }^{+} \mathrm{CD} 3{ }^{-} \mathrm{CD} 25^{+}$subpopulation $^{62}$.

Villus number quantification aimed to demonstrate the health or fragility/destruction of the intestinal mucosa. Our results agree to previous studies, which showed that damage to global architecture and epithelial barrier, consequent to the loss of cell-cell junctional integrity, is a typical reaction caused by inflammatory 
processes $^{63,64}$.

To ascertain, from the architectural point of view, that not only villus number is altered we continued our analysis by assessing villus area and $\mathrm{H} / \mathrm{W}$ ratio. For both parameters, the group that received OVA six hours before P-CD (EXP-1) compares to NEG CONT but not to EXP 2, EXP-3 and POS CONT. These findings can be explained by the changes observed in the height and width of each villus.

A fine balance between the numerous signaling pathways and transcription factors lead to proliferation and differentiation of the intestinal stem cells to epithelial cell types. As cells mature, they migrate to the villus tip where they are shed after apoptosis is triggered ${ }^{65}$. These are crucial for the adequate physiology and mucosal barrier that reinforces intestinal epithelial integrity and architectural maintenance ${ }^{66}$. Absorptive enterocytes, secretory goblet, enterochromaffin and Paneth cells all derive from the intestinal stem cells and play a central role in the different functions of the gastrointestinal tract such as nutrient absorption, avoiding the entry of both pathogenic organisms and large antigenic molecules, innate inflammatory signaling, secretion of molecules that contribute to the mucosal barrier maintenance, antigen presentation to underlying immune cells, and production of endocrine signaling molecules ${ }^{67}$. Inflammatory cytokines disrupt this balance and induce the flattening of the villi as observed in the EXP 2, EXP 3 and POS CONT groups. As the villi become wider and shorter the absorption diminishes as the number of villi per area also diminishes per mm ${ }^{2}$. Animals in these three groups reduced food intake and presented weight loss (data not shown). Villus area is a parameter still poorly described in the literature. Studies from our group have already proposed that this is an important aspect to be assessed since the identification of the occurrence of histological adaptation may indicate a change in the absorption surface of the small intestine ${ }^{31,32,68}$.

Serological and histological data correlate well in our experimental model. EXP-1 that received OVA six hours prior to P-CD has low antibody titers and normal villus morphology permitting us to conclude that there was an induction of tolerance to OVA (the novel food protein). EXP-2 and EXP-3 received the novel food protein, at or after the onset of the peanut challenge diet respectively and were challenged with both a subcutaneous injection of OVA and O-CD presented both high antibody titers and morphological alterations of the gut. These results are in line with those found by other studies carried out in our research group ${ }^{27,39}$. The same was observed in the villus IEC/IEL ratio, where EXP-2, EXP-3 and POS CONT showed significant lower ratios than NEG CONT and EXP-1. This IEC/IEL ratio reduction occurs due to increased proliferation of leucocytes in the lamina propria and massive infiltration in epithelium as already observed ${ }^{69-71}$.

Jejunum swiss rolls showed increased leucocyte infiltration in EXP-2, EXP-3 and POS CONT when compared to NEG CONT, i.e., one has a greater chance to present altered jejunum mucosa when the duodenum is also altered. This behavior was already verified by $\mathrm{us}^{31}$ and by others in the literature ${ }^{72}$.

Although it was possible to better understand the timing between the introduction of an allergenic food with new food proteins, other studies using models of allergy and inflammation still need to be carried out. Here we confirm again that feeding a novel food protein in a physiological context of the gut mucosa induces tolerance to this protein even if shortly afterwards an inflammatory insult occurs. However, the introduction of a novel protein concomitant or after the initiation of an inflammatory process tends to induce food allergy. In conclusion, multiple food allergies may be the consequence of new food proteins introduction during a gut inflammation due to a prior food allergy, as frequently occurs in pediatric scenarios.

\section{References}

1. Seth D, Poowutikul P, Pansare M, Kamat D. Food Allergy: A Review.Pediatr Ann. 2020;49(1):e50-e58.

2. Schulke S, Albrecht M. Mouse Models for Food Allergies: Where Do We Stand? Cells. 2019;8(6).

3. Skypala IJ. Food-Induced Anaphylaxis: Role of Hidden Allergens and Cofactors. Frontiers in immunology. 2019;10:673.

4. Costa C, Coimbra A, Vitor A, Aguiar R, Ferreira AL, Todo-Bom A. Food allergy-From food avoidance to active treatment. Scandinavian journal of immunology. 2020;91(1):e12824. 
5. Gunawardana NC, Durham SR. New approaches to allergen immunotherapy.Annals of allergy, asthma $\&$ immunology : official publication of the American College of Allergy, Asthma, ES Immunology.2018;121(3):293-305.

6. Mahdavinia M. Food Allergy in Adults: Presentations, Evaluation, and Treatment. Med Clin North Am. 2020;104(1):145-155.

7. Popov TA, Mustakov TB, Kralimarkova TZ. Food allergy in adults in Europe: what can we learn from geographical differences? Current opinion in allergy and clinical immunology. 2020.

8. Bender BG. How Do We Address Food Allergies in High-Risk Populations? The journal of allergy and clinical immunology In practice.2020;8(1):339.

9. Feng C, Kim JH. Beyond Avoidance: the Psychosocial Impact of Food Allergies. Clinical reviews in allergy Es immunology.2019;57(1):74-82.

10. Thornqvist V, Middelveld R, Wai HM, et al. Health-related quality of life worsens by school age amongst children with food allergy. Clinical and translational allergy. 2019;9:10.

11. Tang MLK, Lozinsky AC, Loke P. Peanut Oral Immunotherapy: State of the Art. Immunol Allergy Clin North Am. 2020;40(1):97-110.

12. Vazquez-Cortes S, Jaqueti P, Arasi S, Machinena A, Alvaro-Lozano M, Fernandez-Rivas M. Safety of Food Oral Immunotherapy: What We Know, and What We Need to Learn. Immunol Allergy Clin North Am.2020;40(1):111-133.

13. Nurmatov U, Dhami S, Arasi S, et al. Allergen immunotherapy for IgE-mediated food allergy: a systematic review and meta-analysis.Allergy. 2017;72(8):1133-1147.

14. Nowak-Wegrzyn A, Berin MC, Mehr S. Food Protein-Induced Enterocolitis Syndrome. The journal of allergy and clinical immunology In practice. 2020;8(1):24-35.

15. Senba S, Tsuji T, Kikuchi R, et al. Oral mite anaphylaxis after ingestion of Korean pancake. Respir Med Case Rep. 2020;30:101026.

16. Arkwright PD, MacMahon J, Koplin J, et al. Severity and threshold of peanut reactivity during hospitalbased open oral food challenges: An international multicenter survey. Pediatric allergy and immunology : official publication of the European Society of Pediatric Allergy and Immunology. 2018;29(7):754-761.

17. Birot S, Crepet A, Remington BC, et al. Frequentist and Bayesian approaches for food allergen risk assessment: risk outcome and uncertainty comparisons. Scientific reports. 2019;9(1):18206.

18. Spychaj A, Pospiech E, Iwanska E, Montowska M. Detection of allergenic additives in processed meat products. J Sci Food Agric. 2018;98(13):4807-4815.

19. Jedrusek-Golinska A, Piasecka-Kwiatkowska D, Zielinska P, Zielinska-Dawidziak M, Szymandera-Buszka K, Hes M. Soy Preparations Are Potentially Dangerous Factors in the Course of a Food Allergy.Foods. $2019 ; 8(12)$.

20. Arasi S, Castagnoli R, Pajno GB. Oral immunotherapy in pediatrics.Pediatric allergy and immunology: official publication of the European Society of Pediatric Allergy and Immunology. 2020;31 Suppl 24:51-53.

21. Bartnikas LM, Huffaker MF, Sheehan WJ, et al. Racial and socioeconomic differences in school peanutfree policies. The journal of allergy and clinical immunology In practice.2020;8(1):340-342 e341.

22. Taylor SL, Baumert JL. Worldwide food allergy labeling and detection of allergens in processed foods. Chem Immunol Allergy.2015;101:227-234.

23. Larsen JM, Bogh KL. Animal models of allergen-specific immunotherapy in food allergy: Overview and opportunities. Clin Exp Allergy.2018;48(10):1255-1274. 
24. Kreuter R, Wankell M, Ahlenstiel G, Hebbard L. The role of obesity in inflammatory bowel disease. Biochim Biophys Acta Mol Basis Dis. 2019;1865(1):63-72.

25. Kilkenny C, Browne WJ, Cuthill IC, Emerson M, Altman DG. Improving bioscience research reporting: the ARRIVE guidelines for reporting animal research. PLoS biology. 2010;8(6):1-5.

26. Reeves PG, Nielsen FH, Fahey GC, Jr. AIN-93 purified diets for laboratory rodents: final report of the American Institute of Nutrition ad hoc writing committee on the reformulation of the AIN-76A rodent diet. The Journal of nutrition. 1993;123(11):1939-1951.

27. Campos SM, de Oliveira VL, Lessa L, et al. Maternal immunomodulation of the offspring's immunological system. Immunobiology.2014;219(11):813-821.

28. Teixeira G, Paschoal PO, de Oliveira VL, et al. Diet selection in immunologically manipulated mice. Immunobiology.2008;213(1):1-12.

29. Mirotti L, Mucida D, de Sa-Rocha LC, Costa-Pinto FA, Russo M. Food aversion: a critical balance between allergen-specific IgE levels and taste preference. Brain, behavior, and immunity.2010;24(3):370-375.

30. Costa-Pinto FA, Basso AS. Neural and behavioral correlates of food allergy. Chem Immunol Allergy. 2012;98:222-239.

31. Pereira ESA, Lourenco AL, Marmello BO, Bitteti M, Teixeira G. Comparison of two techniques for a comprehensive gut histopathological analysis: Swiss Roll versus Intestine Strips. Exp Mol Pathol.2019;111:104302.

32. Pereira e Silva A, Soares JRA, Mattos EBA, et al. A histomorphometric classification system for normal and inflamed mouse duodenum-Quali-quantitative approach. International journal of experimental pathology. 2018;00:1-10.

33. Ahmad I, Rehman A, Khan JA, et al. Effects of varying local temperature on the optical properties of cells in-vitro.Photodiagnosis Photodyn Ther. 2015;12(3):459-465.

34. De Martinis M, Sirufo MM, Suppa M, Ginaldi L. New Perspectives in Food Allergy. International journal of molecular sciences.2020;21(4).

35. Lodge CJ, Allen KJ, Lowe AJ, Dharmage SC. Overview of evidence in prevention and aetiology of food allergy: a review of systematic reviews. International journal of environmental research and public health. 2013;10(11):5781-5806.

36. Goncalves LC, Guimaraes TC, Silva RM, et al. Prevalence of food allergy in infants and pre-schoolers in Brazil. Allergologia et immunopathologia. 2016;44(6):497-503.

37. Gupta RS, Warren CM, Smith BM, et al. Prevalence and Severity of Food Allergies Among US Adults. JAMA Netw Open.2019;2(1):e185630.

38. Abrams EM, Sicherer SH. Diagnosis and management of food allergy.CMAJ. 2016;188(15):1087-1093.

39. Paschoal PO, Campos SM, Pedruzzi MM, et al. Food allergy/hypersensitivity: antigenicity or timing? Immunobiology.2009;214(4):269-278.

40. Pereira e Silva A, Campos SM, Pedruzzi MM, et al. Reestablishment of the Physiologic Tolerogenic Milieu after a Gut Inflammation is Time Dependent. Austin Immunology. 2016;1(2).

41. Tordesillas L, Berin MC. Mechanisms of Oral Tolerance.Clinical reviews in allergy 85 immunology. 2018;55(2):107-117.

42. Wang HY, Li Y, Li JJ, et al. Serological investigation of IgG and IgE antibodies against food antigens in patients with inflammatory bowel disease. World J Clin Cases. 2019;7(16):2189-2203.

43. Shakoor Z, AlFaifi A, AlAmro B, AlTawil LN, AlOhaly RY. Prevalence of IgG-mediated food intolerance among patients with allergic symptoms.Ann Saudi Med. 2016;36(6):386-390. 
44. Lee E, Barnes EH, Mehr S, Campbell DE. Differentiating Acute Food Protein-Induced Enterocolitis Syndrome From Its Mimics: A Comparison of Clinical Features and Routine Laboratory Biomarkers. The journal of allergy and clinical immunology In practice. 2019;7(2):471-478 e473.

45. Mehr S, Campbell DE. Food protein-induced enterocolitis syndrome: guidelines summary and practice recommendations. Med J Aust.2019;210(2):94-99.

46. Johansson SG, Hourihane JO, Bousquet J, et al. A revised nomenclature for allergy. An EAACI position statement from the EAACI nomenclature task force. Allergy. 2001;56(9):813-824.

47. Muraro A, Werfel T, Hoffmann-Sommergruber K, et al. EAACI food allergy and anaphylaxis guidelines: diagnosis and management of food allergy. Allergy. 2014;69(8):1008-1025.

48. Morita H, Nomura I, Matsuda A, Saito H, Matsumoto K. Gastrointestinal food allergy in infants. Allergology international : official journal of the Japanese Society of Allergology. 2013;62(3):297-307.

49. Nowak-Wegrzyn A, Katz Y, Mehr SS, Koletzko S. Non-IgE-mediated gastrointestinal food allergy. The Journal of allergy and clinical immunology. 2015;135(5):1114-1124.

50. Diaferio L, Caimmi D, Verga MC, et al. May Failure to Thrive in Infants Be a Clinical Marker for the Early Diagnosis of Cow's Milk Allergy? Nutrients. 2020;12(2).

51. Kettman J, Skarvall $\mathrm{H}$. The allogeneic effect: bystander effect in the primary immune response in vitro. Eur J Immunol.1974;4(10):641-645.

52. Pacheco Y, Acosta-Ampudia Y, Monsalve DM, Chang C, Gershwin ME, Anaya JM. Bystander activation and autoimmunity. Journal of autoimmunity. 2019;103:102301.

53. Bloch KJ, Walker WA. Effect of locally induced intestinal anaphylaxis on the uptake of a bystander antigen. The Journal of allergy and clinical immunology. 1981;67(4):312-316.

54. Turner MW, Boulton P, Shields JG, et al. Intestinal hypersensitivity reactions in the rat. I. Uptake of intact protein, permeability to sugars and their correlation with mucosal mast-cell activation.Immunology. 1988;63(1):119-124.

55. Korte R, Lepski S, Brockmeyer J. Comprehensive peptide marker identification for the detection of multiple nut allergens using a non-targeted LC-HRMS multi-method. Analytical and bioanalytical chemistry. 2016;408(12):3059-3069.

56. Dahlman-Hoglund A, Dahlgren U, Ahlstedt S, Hanson LA, Telemo E. Bystander suppression of the immune response to human serum albumin in rats fed ovalbumin. Immunology. 1995;86(1):128-133.

57. Backstrom NF, Dahlgren UI. Bystander suppression of collagen-induced arthritis in mice fed ovalbumin. Arthritis Res Ther.2004;6(2):R151-160.

58. Mondoulet L, Dioszeghy V, Puteaux E, et al. Specific epicutaneous immunotherapy prevents sensitization to new allergens in a murine model. The Journal of allergy and clinical immunology.2015;135(6):1546-1557 e1544.

59. Mondoulet L, Dioszeghy V, Busato F, et al. Gata3 hypermethylation and Foxp3 hypomethylation are associated with sustained protection and bystander effect following epicutaneous immunotherapy in peanutsensitized mice. Allergy. 2019;74(1):152-164.

60. Oyoshi MK, Oettgen HC, Chatila TA, Geha RS, Bryce PJ. Food allergy: Insights into etiology, prevention, and treatment provided by murine models. The Journal of allergy and clinical immunology.2014;133(2):309-317.

61. Bacher $\mathrm{P}$, Scheffold $\mathrm{A}$. The effect of regulatory $\mathrm{T}$ cells on tolerance to airborne allergens and allergen immunotherapy. J Allergy Clin Immunol. 2018;142(6):1697-1709. 
62. Amu S, Brisslert M. Phenotype and function of CD25-expressing B lymphocytes isolated from human umbilical cord blood. Clinical \& developmental immunology. 2011;2011:481948.

63. Jarmakiewicz-Czaja S, Piatek D, Filip R. The Influence of Nutrients on Inflammatory Bowel Diseases. J Nutr Metab. 2020;2020:2894169.

64. Burns G, Pryor J, Holtmann G, Walker MM, Talley NJ, Keely S. Immune Activation in Functional Gastrointestinal Disorders. Gastroenterol Hepatol (N Y). 2019;15(10):539-548.

65. Thompson CA, DeLaForest A, Battle MA. Patterning the gastrointestinal epithelium to confer regionalspecific functions. Dev Biol. 2018;435(2):97-108.

66. Gassler N. Paneth cells in intestinal physiology and pathophysiology. World J Gastrointest Pathophysiol.2017;8(4):150-160.

67. Farre R, Fiorani M, Abdu Rahiman S, Matteoli G. Intestinal Permeability, Inflammation and the Role of Nutrients. Nutrients.2020;12(4).

68. Antunes DM, da Costa JP, Campos SM, et al. The serum D-xylose test as a useful tool to identify malabsorption in rats with antigen specific gut inflammatory reaction. International journal of experimental pathology. 2009;90(2):141-147.

69. Liu T, Navarro S, Lopata AL. Current advances of murine models for food allergy. Molecular immunology. 2016;70:104-117.

70. Ma WT, Gao F, Gu K, Chen DK. The Role of Monocytes and Macrophages in Autoimmune Diseases: A Comprehensive Review. Frontiers in immunology. 2019;10:1140.

71. Samarani S, Sagala P, Jantchou P, et al. Phenotypic and Functional Changes in Peripheral Blood Natural Killer Cells in Crohn Disease Patients. Mediators of inflammation. 2020;2020:6401969.

72. Davis JS, Gupta V, Gagea M, Wu X. An Advanced Histologic Method for Evaluation of Intestinal Adenomas in Mice Using Digital Slides.PloS one. 2016;11(3):e0151463.

Figure Legends

Figure 1 - Timeline of experimental protocol. PPE: Peanut Protein Extract; OVA: Ovalbumin; P-CD: Peanut challenge diet; O-CD: OVA challenge diet.

Figure 2-A : Mean group anti-peanuts IgG titers. All groups immunized with PPE showed significant higher titers when compared to NEG CONT, which was immunized with saline. POS CONT and EXP-1 presented lower IgG titers at the end of the experiment, while EXP-2 and EXP-3 maintained high IgG titers throughout the protocol: B : Mean Anti-OVA IgG titers +- SD per group after: primary inoculation with OVA: NEG CONT showed significant lower titers than EXP-2, EXP-3 and POS CONT; post OVA booster: NEG CONT and EXP-1 showed significant lower titers than EXP-2, EXP-3 and POS CONT; and post O-CD: NEG CONT and EXP-1 showed significant lower titers than EXP-2, EXP-3 and POS CONT.

Figure 3 - A : Mean percentage of $\mathrm{TCD}^{+}{ }^{+}$and $\mathrm{TCD} 8^{+}$cells in the plot $+-\mathrm{SD}$ per group. No significant differences were observed in TCD $4^{+}$cells, but NEG CONT and EXP-1 showed a significant lower percentage of TCD $8^{+}$cells than the other groups; B : Mean group percentage of TCD $4^{+} \mathrm{CD} 25^{+}$Foxp $3^{+}$cells from the $\mathrm{TCD}^{+}$gate $+-\mathrm{SD}$. NEG CONT and EXP-1 showed significant higher percentages when compared to EXP2, EXP-3 and POS CONT; C : Mean group percentage of effector B-B220 ${ }^{+} \mathrm{CD} 3{ }^{-} \mathrm{CD} 25^{+}$cells $+-\mathrm{SD}$. NEG CONT and EXP-1 had a significantly smaller population compared to EXP-3 and POS CONT. We did not observe significant differences between groups EXP-1 and EXP-2.

Figure 4 - Digital slide representative of each group showing general morphology of duodenum villi.

Figure 5 - Digital slide representative of each group showing general morphology of jejunum villi. The arrows show inflammatory cells in the epithelial layer, which can be an inflammatory marker. 
Figure S1 - Mean caloric intake per gram of body weight per cage during OVA challenge diet +- SD per group. NEG CONT and EXP-1 showed a significant higher consumption than all other groups.

Figure S2 - A : Mean group villus number per 8000 $\mu \mathrm{m}$ in the duodenum $\pm \mathrm{SD}$. EXP-1 showed a significant higher villi number than EXP-2, EXP-3 and POS CONT; B : Mean villus area + SD per group. NEG CONT and EXP-1 showed a significant higher area in each villus than the other groups; C : Mean group villus $\mathrm{H} / \mathrm{W}$ ratio + SD. NEG CONT and EXP-1 showed a significant higher $\mathrm{H} / \mathrm{W}$ ratio than the other groups;D : Mean group villus IEC/IEL ratio + SD per group. NEG CONT and EXP-1 showed a significant higher ratio than the other groups;E : Jejunal Mean villus IEC/IEL ratio + SD per group. NEG CONT showed a significant higher ratio than the other groups, except EXP-1.
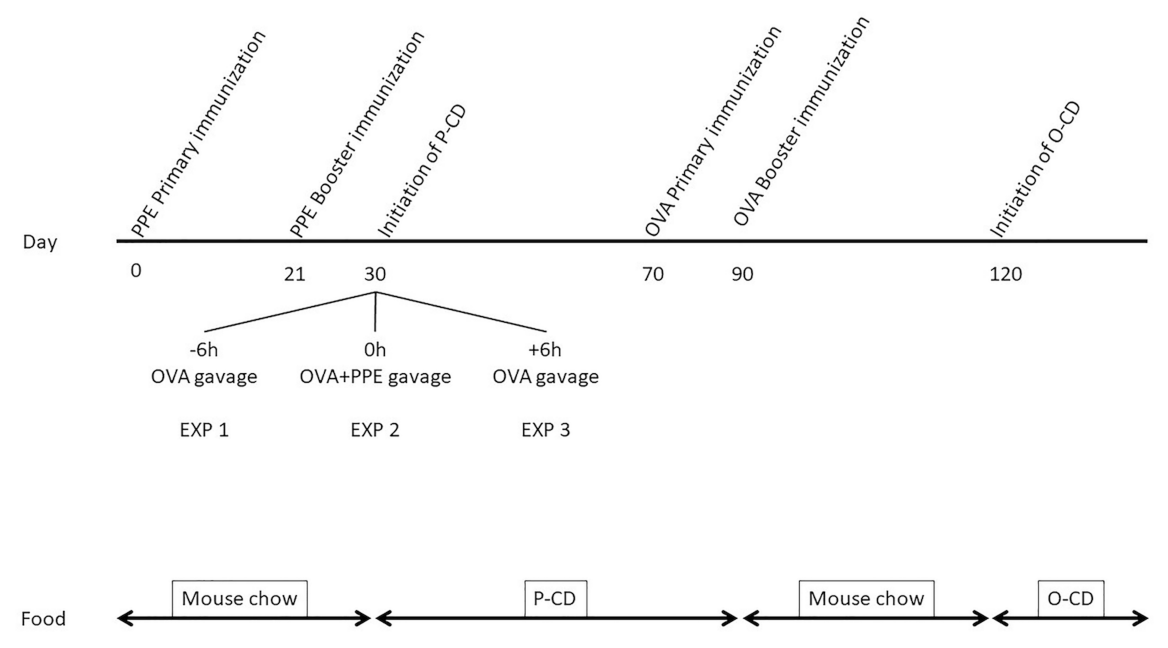
A

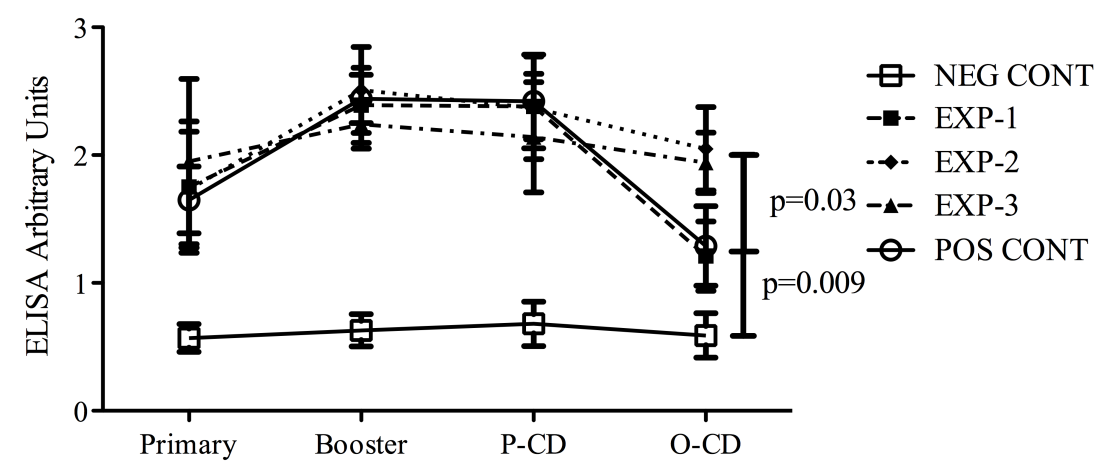

B

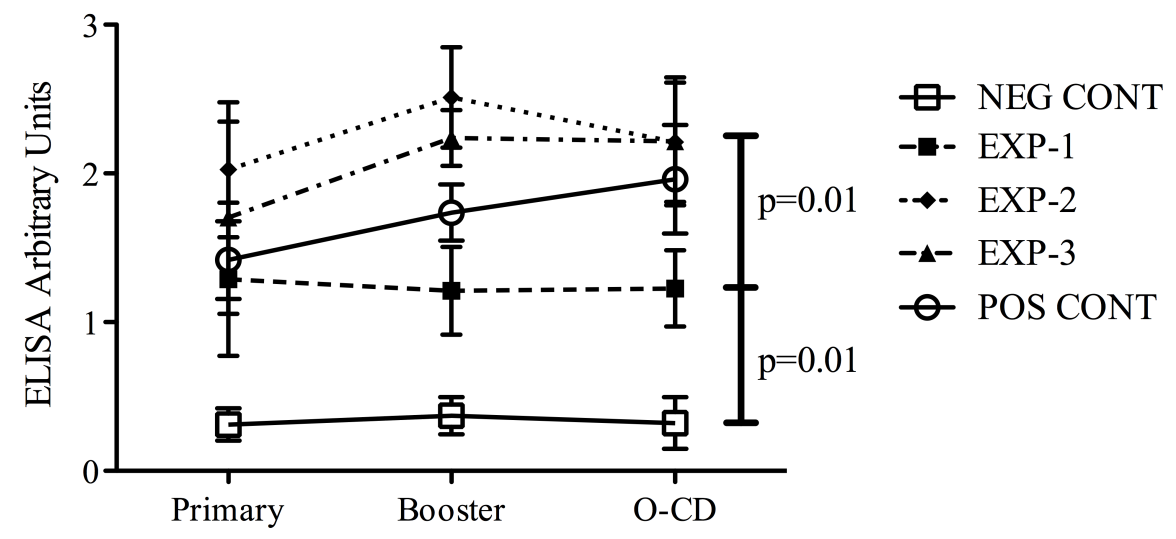



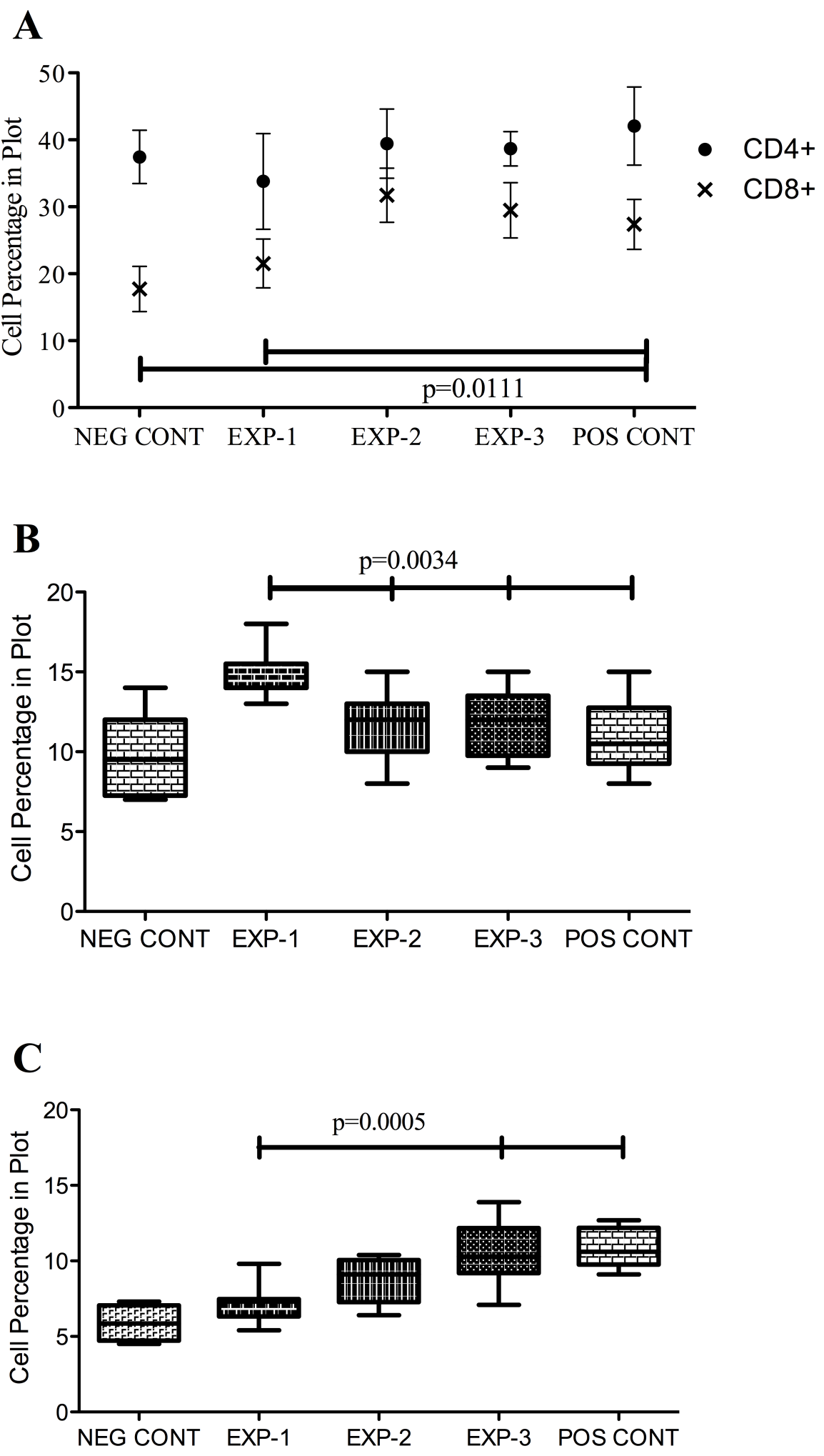

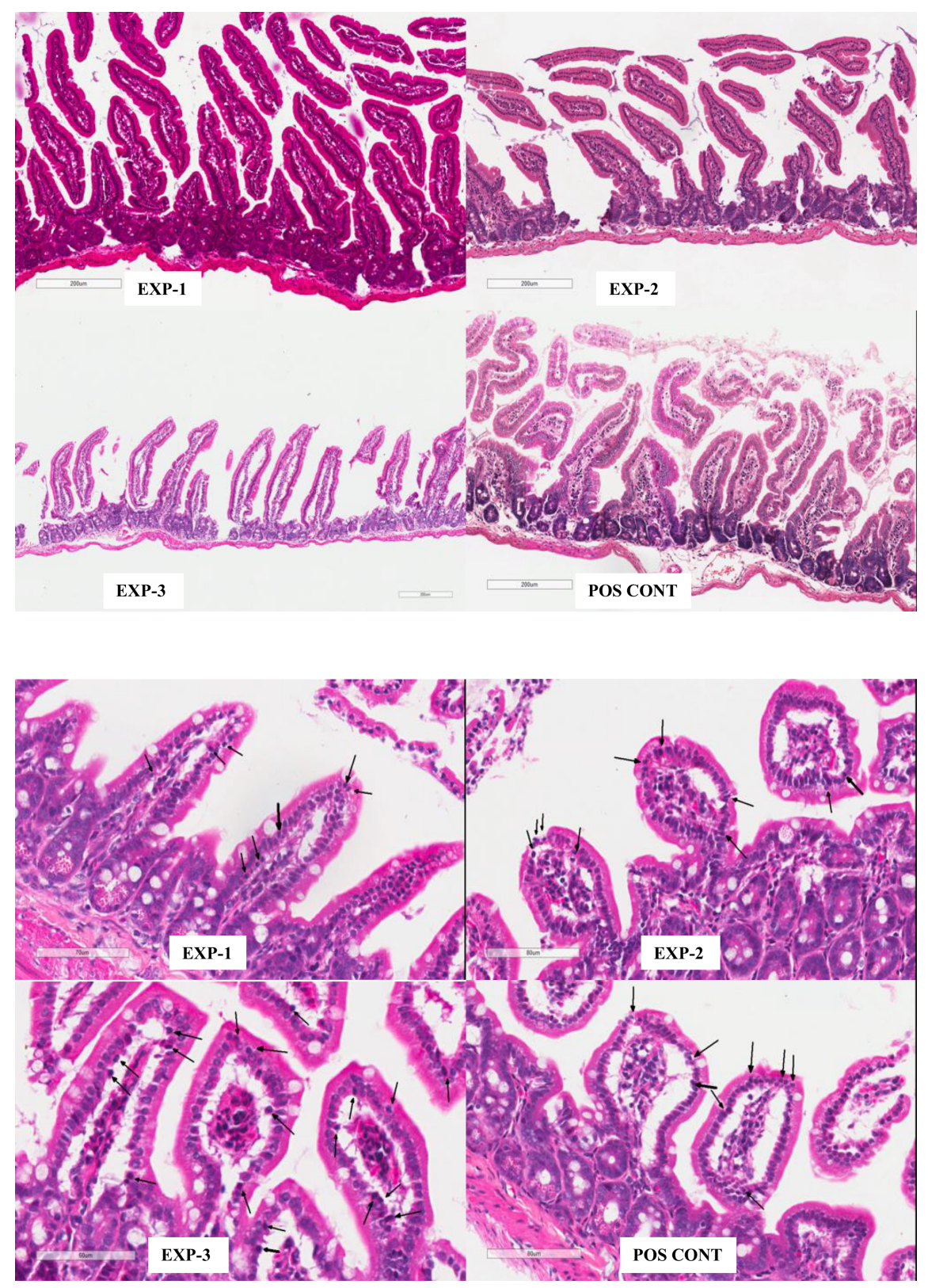